\title{
Ultrasound Measurement of Endometrial Thickness for Detecting Endometrial Malignancy with Uterine Bleeding in Postmenopausal Women- A Systematic Review
}

ALIA IQBAL, SYEDA KHADIJA-TUL-SUGHRA MURRIUM, MARIA YASEEN, KIRAN SHAKEEL, MUHAMMAD ZAFAR IQBAL University Institute of Radiological Sciences and Medical Imaging Technology, Faculty of Allied Health Sciences the University of Lahore, Pakistan Correspondence to Alia Iqbal, E-mail: aliaiqbal746@gmail.com

\begin{abstract}
Background: Post-menopausal Bleeding is extremely doubtful of existence a mark intended for incidence of EC as well as around $5 \%$ to $12 \%$ of post-menopausal bleeding consequences commencing endometrial cancer. EC is greatest communal Gynecologic Malignancy into established countries. Thin endometrium ( $\leq 4 \mathrm{~mm}$ ) devours precise high NPV (99\%). Thickened Endometrium has been considered by way of sign intended for an offensive assessment.

Aim: To determine the ET measurement for detecting endometrial malignancy with uterine bleeding in post-menopausal females. Methods: The search was led according to Systematic Reviews Ethics. Searched databases were: Google scholar and Web Research from January 2014 and November 2020. Around nine studies were designated for this Systemic Review. We mined subsequent facts: Design of study, mean age and range, menopause duration, range and mean, BMI range and mean, as well as ET range and mean.

Conclusion: From beyond examination this review concluded that, the Endometrial Thickness among the suggested alteration in cut off 3-5 mm within repetitive ultrasound practice must be ended in elevation threat females identify for malignancy before in Postmenopausal females as well as ultrasound is attested chosen a convenient implement to diagnose.

Keywords: Post-menopausal Bleeding, Endometrial Thickness, Endometrial Cancer.
\end{abstract}

\section{INTRODUCTION}

Post-menopausal Bleeding is recurrent occasion within postmenopausal females as well as signifies upto $10 \%$ of whole appointments into secluded practice of gynecology. Incidence of Post-menopausal Bleeding within entire post-menopausal females is about $10 \%$, frequently produced through benign results for instance Endometrial Hyperplasia /Atrophy/Benign Bolyps ${ }^{1,2}$. Though, Post-menopausal Bleeding is too extremely doubtful of existence a mark intended for EC presence as well as about $5 \%$ to $12 \%$ of Post-menopausal Bleeding consequences from endometrial cancer ${ }^{3}$. EC is utmost communal Gynecologic Malignancy in established countries, as well as EC is analyzed in $>90 \%$ females with post-menopausal vaginal bleeding ${ }^{4}$. For such post-menopausal females, EC threat arrays from $4.9 \%-11.5 \%$ as well as consequently, initial as well as precise analysis is significant as well as PMB must be correctly examined ${ }^{5}$. Thin endometrium ( $\leq 4 \mathrm{~mm}$ ) devises actual high NPV (99\%) as well as sampling of histologycan be evaded ${ }^{6}$.

The gold-standard intended for clinical search of PMB was association-based Dilatation as well as Curettage in past, nonetheless now a days here are amount of office-based techniques, like Endometrial Biopsy as well as Hysteroscopy, to evaluate females with this criticism as well as Endometrial Biopsy devises nearly totally substituted Dilatation and Curettage. Outpatient Endometrial Biopsy done among Pipelle sample expedient (cooper surgical, CT, trumbull) is modest for performing, comparatively cheap, as well as extremely delicate to detect $\mathrm{EC}^{7,8}$ In current, measurement of sonography of ET comprises too progressively prevalent like noninvasive analytic implement to assess pathology of uterine with PMB in patients. Sampling of endometrium is suggested, among cut-off value $4 \mathrm{~mm}$ or $5 \mathrm{~mm}$, intended for indicative post-menopausal females. Though, diverse strategies utilize diverse cut off value of ET, variable from $3 \mathrm{~mm}$ to $5 \mathrm{~mm}$, to exclude Endometrial Malignancy ${ }^{9-12}$. Widespread utilize of sonography comprises too allow able accompanying ET result into asymptomatic post-menopausal females. Though, it devises been optional that the 4-mm or 5-mm confines usually utilized to exclude Malignancy into symptomatic post-menopausal females are not transportable to asymptomatic patient ${ }^{13,14}$. So, our aim was for assessing analytic value of sonography, slow ET in forecasting

Received on 11-01-2021

Accepted on 17-05-2021
Endometrial Pathologies within post-menopausal females among Vaginal Bleeding as well as in those by asymptomatic thickened endometrium ( $\geq 5 \mathrm{~mm}$ ). For accomplishing this, we examined finest cut-off standards for using to discriminate among post-menopausal females with intra-uterine pathologies as well as those with-out such pathology.

Fig. 1: ET measurement method. Measurement is endedupon a Sagittal Transvaginal Image, assessing as of the anterior endometrial myometrial border to posterior endometrial myometrial border (calipers). This is a doublelayer dimensionas it comprises layers of posterior and anterior endometrium.

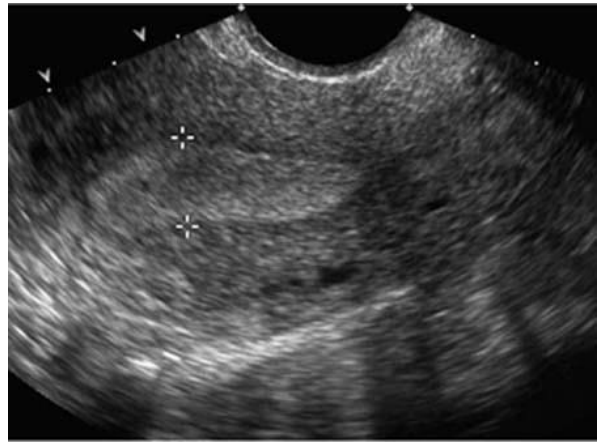

Fig 2: In Secretory Phase at it's thickest, up to $16 \mathrm{~mm} \mathrm{10,} \mathrm{as} \mathrm{well} \mathrm{as}$ develops consistently echo-genic, by way of useful layer becomes Edematous as well as Isoechoic to Basal Layer. There is done broadcast as well as posterior Acoustic improvement noted.

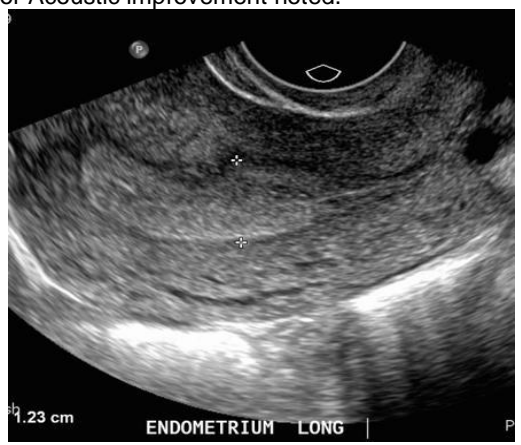




\section{MATERIALS AND METHODS}

Sources of data and searches examined in 2 PubMed as well as science web intended to study report upon ET in post-menopausal females, available amongst Jan 2014 to Nov 2020. English was set as language limit. Terms utilized to search comprised "Endometrial Thickness", "Post-menopausal," as well as "post menopause." E.g., to search in PubMed, we utilized subsequent MeSH Terms: (Endometrial [All fields] AS WELL AS Thickness [all fields] AS WELL AS Asymptomatic [all fields] AS WELL AS ("Postmenopause" [MeSH terms] OR "Post menopause" [all fields]) AS WELL AS post-menopausal [all fields]).

Study selection: One detective mark off title as well as abstract recognized through pursuit to exclude identical as well as unrelated articles. We likewise excepted studies by $<150$ females comprised (this was random standard, fair pointing to obtain additional healthy records), revisions with pre-menopausal and/or peri-menopausal females, also females pleasing TMX, hormonal HRT, Aromatase Inhibitors, or some additional SERM were let off. Formerly, complete edition of the residual article was autonomouslystudiedthrough3 investigators to identify latent entitled revisions founded upon subsequent criteria: study reporting upon endometrial thickness in post-menopausal females.

Data extraction: One investigator independently extracted data for each study ultimately included. We mined subsequent facts: Design of study, mean age and range, menopause duration, range and mean, BMI range and mean, as well as ET range and mean.

\section{DISCUSSION}

In 2014 Bahadir Saatli et al., assessed 530 patients on ET role to detect Endometrial Pathologies within asymptomatic postmenopausal females, in which he evaluated importance of Endometrial sample into asymptomatic, bleeding free postmenopausal females, they devour ET more or equivalent to $5 \mathrm{~mm}$. Transvaginal ultrasonography was performed. About 530 asymptomatic post-menopausal females endured the evaluation of ultrasonography by following sampling of endometrium. The mean age remained 61 (range: $46-82$ ). The mean duration of menopause 16 (range: 1-48). The mean body mean index (BMI) 27.4 (range: 18-48). Mean Endometrial Stripe Thickness was $8.7 \mathrm{~mm}$ (Range: 6 to 26). They concluded that, one hundred and six inquiries were done for detecting 1 adenocarcinoma case. Though this is a high numeral per-case finding of Endometrial Adenocarcinoma, as increasing occurrence of cancer of endometrium, enormous potential prosecutions by replacement conditions intended for asymptomatic females are desirable for investigating the significance of congealed Endometrial Stripe within postmenopausal females ${ }^{15}$.

In 2015 AS-W Wong et.al., assessed 4383 females with post-menopausal bleeding between 2002 and 2013, on review of ET to detect Endometrial Cancer in post-menopausal bleeding by TVUS dimension of endometrial thickness as well as Endometrial Biopsies were founding females offering PMB, histology of endometrium was utilized as mention average for calculating accurateness approximations. In $3.8 \%$ of women endometrial cancer was diagnosed. The mean age is 55 (range: $52-62$ ). The mean duration of menopause 50 (range: 48-52). The mean body of index 23.9 (range: 21.6-26.6). The mean endometrial thickness 15.7 (range: 9.5-23.0). They concluded that, TVUS by $3 \mathrm{~mm}$ cut off compromises elevation sensitivity to detect Endometrial Cancer as well as may detect females by PMB who are extremely improbable for having Endometrial Cancer, thus evading additional aggressive Endometrial Biopsy ${ }^{16}$

In 2017 Amelie Schramm et. al., did a retrospective study in 254 patients on worth of Endometrial Thickness measured through Transvaginal Ultrasound to predict the Endometrial Cancer's people within post-menopausal bleeding. They assessed TVUS extents of ET in patient with postmenopausal bleeding to detect the endometrial carcinoma. The mean age 64 (range: 40-92). The mean body mass index 28 (16.9-103.6). The mean endometrial thickness $>4 \mathrm{~mm}(83.1 \%)$; $>10 \mathrm{~mm}(49.9 \%)$ and $>15 \mathrm{~mm}(28.6 \%)$. They concluded that, tedious usage of ET dimension through transvaginal ultrasound do not elect actual analytic implement for Endometrial cancer since this one devours a low performance of diagnosis within asymptomatic post-menopausal females. Additional forthcoming studies are obligatory for assessing ET measurements among Transvaginal Ultrasound as a broadcast technique within these females ${ }^{17}$.

In 2018 Alper Basbug et al., did a retrospective study in 155 patients showing the association among Adnexal Lesion and Endometrial Thickness in post-menopausal women. They employed trans-vaginal Ultrasonography to evaluate the Adnexal Lesions synchronously perceived thin post-menopausal females by means of augmented Endometrial Thickness. Standard deviation age $57.75 \pm 11.89$. The standard deviation duration of menopause 11.23 \pm 7.65 . The standard deviation body mass index $33.60 \pm 3.90$. The standard deviation endometrial thickness $11.73 \pm 5.97$. They concluded that, within post-menopausal females, Adnexal Lesions may be perceived concurrently by augmented Endometrial Thickness. Incidentally, Transvaginal Ultrasound deals significant prospects to evaluate both Endometrium as well as Adnexa ${ }^{17}$.

In 2019 Magdalena Pirog et al did a retrospective study in 57 patients on thin red line post-menopausal unusual uterine-bleeding with Endometrial Thickness fewer that $4 \mathrm{~mm}$. They evaluated threat of Endometrial Malignancy within post-menopausal females among AUB among ET $\leq 4 \mathrm{~mm}$. The standard deviation age 56.75 \pm 6.6 . The standard deviation duration of menopause $51.1 \pm 4.3$. The mean body mass index 25.3 (range: 23.5-29.4). The standard deviation endometrial thickness $3.2 \pm 0.6$. They concluded that, Postmenopausal women with abnormal uterine bleed as well as Endometrial Echo $\leq 4 \mathrm{~mm}$ are less probable for having malignant situation. Though, part of assessment of histology cannot remain destabilized, particularly within females on elevated threat of endometrial cancer, as well as tedious Endometrial Biopsy must be measured. We commend a alteration in cut off $2 \mathrm{~mm}$ in usual repetition ${ }^{18}$.

In 2020 Dinesh Palipana et.al., did a retroactive study in 222 people investigating women by postmenopausal bleeding. The mean age 60.9 (range: 59.7-62.2). The mean duration of menopause 31.9. The range body mass index 30.6-33.1. The range endometrial thickness $>4 \mathrm{~mm}$ (7.4-9.1) and $>10 \mathrm{~mm}$ (10.917.6). They concluded that, frontier of $3 \mathrm{~mm}$ intended for endometrial thicknesswithin postmenopausal bleeding, accompanied by office Endometrial Biopsy, must be deliberated for ensuringappropriateanalysis ${ }^{19}$.

\section{CONCLUSION}

From beyond examination this review concluded that, the Endometrial Thickness among the suggested alteration in cut off3$5 \mathrm{~mm}$ within repetitive ultrasound practice must be ended in elevation threat females identify for malignancy before in Postmenopausal females as well as ultrasound is attested chosen a convenient implement to diagnose.

Conflict of Interest: There is no conflict of interest

\section{REFERENCES}

1. Schramm A, Ebner F, Bauer E, Janni W, Friebe-Hoffmann U, Pellegrino $\mathrm{M}$, et al. Value of endometrial thickness assessed by transvaginal ultrasound for the prediction of endometrial cancer in patients with postmenopausal bleeding. Archives of Gynecology and Obstetrics. 2017;296(2):319-26.

2. Carugno J. Clinical management of vaginal bleeding in postmenopausal women. Climacteric. 2020:1-7.

3. Dueholm M, Hjorth I, Dahl K, Pedersen L, Ørtoft G. Identification of endometrial cancers and atypical hyperplasia: development and validation of a simplified system for ultrasound scoring of endometrial pattern. Maturitas. 2019;123:15-24.

4. Saso S, Chatterjee J, Georgiou E, Ditri AM, Smith JR, GhaemMaghami S. Endometrial cancer. Bmj. 2011;343. 
5. Seckin B, Cicek MN, Dikmen AU, Bostancı El, Muftuoglu $\mathrm{KH}$ Diagnostic value of sonography for detecting endometrial pathologies in postmenopausal women with and without bleeding. Journal of Clinical Ultrasound. 2016;44(6):339-46.

6. Fakhar S, Saeed G, Khan AH, Alam AY. Validity of pipelle endometria sampling in patients with abnormal uterine bleeding. Annals of Saudi medicine. 2008;28(3):188-91.

7. Abdelazim IA, Abdelrazak KM, Elbiaa AA, Al-Kadi M, Yehia $A H$. Accuracy of endometrial sampling compared to conventional dilatation and curettage in women with abnormal uterine bleeding. Archives of gynecology and obstetrics. 2015;291(5):1121-6.

8. Malanowska-Stega Z, Delpriore G. Simultaneous multiple method outpatient uterus biopsy device and method. Google Patents; 2020.

9. Yasa C, Dural O, Bastu E, Ugurlucan FG, Nehir A, İyibozkurt AC. Evaluation of the diagnostic role of transvaginal ultrasound measurements of endometrial thickness to detect endometria malignancy in asymptomatic postmenopausal women. Archives of gynecology and obstetrics. 2016;294(2):311-6.

10. Van Hanegem N, Breijer M, Khan K, Clark T, Burger M, Mol B, et al. Diagnostic evaluation of the endometrium in postmenopausal bleeding: an evidence-based approach. Maturitas. 2011;68(2):155-64.

11. Patel V, Wilkinson EJ, Chamala S, Lu X, Castagno J, Rush D. Endometrial thickness as measured by transvaginal ultrasound and the corresponding histopathologic diagnosis in women with postmenopausal bleeding. International Journal of Gynecologica Pathology. 2017;36(4):348-55.

12. Wolfman W, Leyland N, Heywood M, Singh SS, Rittenberg DA, Soucy $\mathrm{R}$, et al. Asymptomatic endometrial thickening. Journal of Obstetrics and Gynaecology Canada. 2010;32(10):990-9.
13. Carolin Solomi V. A Prospective Observational Study to Determine the Aetiology of Postmenopausal Bleeding and Correlation of Endometrial Thickness in Endometrial Carcinoma in Our Population: Christian Medical College, Vellore; 2017.

14. Liberis V, Tsikouras P, Christos Z, Ammari A, Dislian V, Koutlaki N, et al. The contribution of hysteroscopy to the detection malignancy in symptomatic postmenopausal women. Minimally Invasive Therapy \& Allied Technologies. 2010;19(2):83-93.

15. aatli B, Yildirim N, Olgan S, Koyuncuoglu M, Emekci O, Saygılı U. The role of endometrial thickness for detecting endometrial pathologies in asymptomatic postmenopausal women. Australian and New Zealand Journal of Obstetrics and Gynaecology. 2014;54(1):36-40.

16. Wong $\mathrm{AW}$, Lao $\mathrm{TH}$, Cheung $\mathrm{C}$, Yeung $\mathrm{S}$, Fan $\mathrm{H}, \mathrm{Ng} \mathrm{P}$, et al. Reappraisal of endometrial thickness for the detection of endometrial cancer in postmenopausal bleeding: a retrospective cohort study. BJOG: An International Journal of Obstetrics \& Gynaecology. 2016;123(3):439-46

17. Basbug A, Dogan O, Yassa M, Pulatoglu C, Kaya AE, Caliskan E. Relationship between adnexal mass and endometrial thickness in postmenopausal period. Medicine. 2018;7(4):848-51.

18. Piróg M, Kacalska-Janssen $O$, Bereza T, Jach $R$. The thin red linepostmenopausal abnormal uterine bleeding with endometrial thickness less than $4 \mathrm{~mm}$. Contemporary Oncology. 2019;23(1):43.

19. Palipana D, Fomin I, Russell E, Chan B, Wong C, Chan E, et al. Investigating women with postmenopausal bleeding: The utility of endometrial thickness in transvaginal ultrasound. Australian and New Zealand Journal of Obstetrics and Gynaecology. 2020. 